Artículos / Articles

\title{
Ecofeminismos y variedades de ambientalismos: Estado de la cuestión
}

\author{
Ecofeminisms and varieties of environmentalisms: State of the \\ matter
}

\author{
M. Eugenia Mediavilla \\ Universidad Pablo de Olavide, España. \\ memedsos@acu.upo.es
}

\section{José M. Echavarren*}

Universidad Pablo de Olavide, España.

jmechavarren@upo.es

Recibido / Received: 13/07/2020 Aceptado / Accepted: 01/11/2020

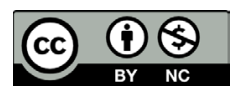

\section{RESUMEN}

Dentro del contexto actual de surgimiento de nuevas formas de activismo ambiental, en el marco de un preocupante escenario de incertidumbre global, este artículo expone las principales teorías desde las que se puede abordar el estudio del ecologismo, sus discursos y sus prácticas, en relación con las diversas manifestaciones de ambientalismos y ecofeminismos. Se encuentra que los ecofeminismos tienen un especial afán narrativo para incorporar la necesidad de los cuidados en las reivindicaciones de los movimientos ambientalistas. Esta propuesta nace en las praxis sociales y territoriales y se va posicionando de manera cada vez más legitimada en investigaciones académicas en el ámbito político y en el debate público internacional. Indagar en las diferentes articulaciones entre ecologismos y feminismos nos permite acercarnos a los usos y apropiaciones de los ecofeminismos, activando nuestra capacidad crítica y de abstracción para imaginar y construir horizontes diferentes, posibles y deseables.

Palabras clave: Ambientalismo hegemónico; ambientalismo crítico; ecología política; crisis socioambiental; sostenibilidad de la vida.

\section{ABSTRACT}

Within the current context of the emergence of new forms of environmental activism in a worrying scenario of global uncertainty, this article sets out the main theories to approach the study of environmentalism, its discourses and its practices, in relation to different kinds of environmentalisms and ecofeminisms. It is found that ecofeminisms have a special narrative desire emphasizing the need for cares in the environmental movement claims. This proposal was born in social and local praxis but spread out to other areas becoming more frequent and legitimized in academic research, in politics and in the international public agenda. Looking at the different links between environmentalism and feminisms offers an opportunity to approach the several uses and appropriations of ecofeminisms, enabling our capacity for abstraction and criticism to imagine and build different, possible and desirable horizons.

Keywords: Hegemonic environmentalism; critical environmental movement; political ecology; environmental crisis; sustainability of life.

* Autor para correspondencia/ Corresponding author: José M. Echavarren, jmechavarren@upo.es

Sugerencia de cita / Suggested citation: Mediavilla, E. y Echavarren, J. (2021). Ecofeminismos y variedades de ambientalismos: Estado de la cuestión. Revista Española de Sociología, 30 (1), a12. https://doi.org/10.22325/fes/res.2021.12 


\section{INTRODUCCIÓN}

Las últimas tres décadas han sido las más calurosas desde el año 1850 y siete de los años más cálidos de los que se tenga registro han tenido lugar en la última década (Intergovernmental Panel on Climate Change [IPCC], 2014). En el actual contexto de crisis socioambiental, que aumenta la probabilidad de ocurrencia de eventos sanitarios y climáticos extremos y las consecuencias en pérdidas de vidas humanas y en deterioro medioambiental, los colectivos ambientalistas aparecen como importantes articuladores entre una diversidad de actores con diferentes grados de responsabilidad en el problema y en su capacidad de trabajo para resolverlo, provenientes de la política, la comunidad científica, el sector empresarial, la población activista y la sociedad civil.

Existen diferentes sensibilidades ambientalistas asociadas a un conjunto de formas de organización y acción en el contexto de crisis climática y socioambiental que atravesamos desde hace varios años. Algunas de estas formas de acción decaen y otras emergen de la mano de movimientos sociales vinculados con activismos ecologistas que intentan, si no dar respuestas, sí ampliar el horizonte de preguntas relacionadas con la sostenibilidad para que no quede reducida la cuestión ambiental a las definiciones que se elaboran dentro los marcos institucionales de organismos internacionales. Nuevas iniciativas ecologistas aparecen para responder a viejos problemas socioambientales con una renovada vitalidad dentro de los marcos teóricos del movimiento de justicia ambiental, la ecología política y los ecofeminismos.

Por su parte, el feminismo ha tenido un auge creciente en este siglo siendo uno de los movimientos sociales más relevantes de la historia reciente. Avanzando en conquistas sociales y denunciando que aún faltan muchas cuestiones por resolver más allá de lograr la igualdad de género, es un movimiento que trabaja en el ámbito de la vida cotidiana y de lo concreto, interpelando los cuerpos y sensibilidades, a diferencia de otros movimientos sociales que se desarrollan en el terreno de la abstracción (Herrero, 2016) o la nostalgia.

Se puede pensar que dos movimientos con objetivos compartidos y con recorridos de larga trayectoria como los ambientalismos y los feminismos podrían trabajar de manera conjunta y complementaria en la lucha por un ambiente sano y justo socialmente. No obstante, hablar de movimientos unívocos y homogéneos implicaría caer en un reduccionismo difícilmente salvable. Existen variedad de ambientalismos y variedad de feminismos que ni entre sus corrientes al interior de cada movimiento logran consensos y en cierto modo, no está mal que eso ocurra.

Entendemos que no siempre será viable el encuentro entre ambientalismos y feminismos. Dado que los grupos ambientalistas no actúan ni piensan de igual forma y tienen diferentes lenguajes de valoración respecto del uso que se hace de la naturaleza (Martínez Alier, 2006, p. 15), variará la manera de abordar la cuestión ambiental que puede ser desde un punto de vista con eje en lo ambiental, lo económico o lo social, y además, con o sin perspectiva de género. Incluso en un mismo grupo ambientalista existen tensiones entre discursos y prácticas al interior aun cuando las personas que lo integran se autoperciben institucionalmente como ecofeministas (Agarwal, 1992; Puleo, 2002).

Para trazar puentes entre ambientalismos y feminismos vemos necesaria la fundamentación, sobre todo teórica, del aporte que los ecofeminismos pueden hacer a cierto tipo de ambientalismo porque los ecofeminismos, producto de sus praxis eco-territoriales, comprenden la ecodependencia y la interdependencia de las formas de reproducción de la vida (Federici, 2017) y, a partir de esos conceptos, construyen el significado de la sostenibilidad. Esta fundamentación teórica es la hoja de ruta que ha tenido el presente trabajo, el cual está basado en la exposición del conjunto de las principales teorías 
desde las que se puede abordar el estudio de los movimientos ambientalistas en relación con sus variedades y con el feminismo, centrando la atención en aquellas perspectivas teóricas enmarcadas en la ecología política (Alimonda, 2006; Martínez Alier, 2006) y en los ecofeminismos (Mellor, 1997; Warren, 1996).

El artículo está organizado en tres secciones. En la primera sección se aborda el conjunto de antecedentes sobre el tema investigado, describiendo ideas provenientes de autores y autoras que han explorado las probabilidades de encuentro entre ambientalismos y feminismos. Seguidamente se describen las variedades teóricas de ambientalismos en el movimiento ecologista presentando dos grandes bloques que agrupan la cuestión ambiental: el ambientalismo hegemónico y el ambientalismo crítico (D'Amico y Agoglia, 2019). Dentro de cada bloque hay corrientes teóricas que ponen el acento en las variables económica/institucional o estructural/sistémica, que están acompañadas por diversos tipos de ecofeminismos y tendrán propuestas diferentes. Y finalmente se expone la importancia de los ecofeminismos, que gracias a su diversidad, pueden plantearse como una narrativa necesaria para las prácticas de cuidados socioambientales que los movimientos ambientalistas van incorporando con mayor intensidad con el paso del tiempo.

\section{ENCUENTROS ENTRE AMBIENTALISMOS Y ECOFEMINISMOS}

Los movimientos ambientalistas en ocasiones funcionan como espacio para encontrar ideas y prácticas feministas, esto es, pueden permitir a aquellas personas que no se autoperciben como feministas encontrar tal ideario sin tener que ir directamente a un espacio feminista (Connell, 1990, p. 452). No obstante no es posible generalizar dado que existen diferentes corrientes al interior del ambientalismo que, si bien pueden compartir el objetivo general de la defensa del medio ambiente, sus estrategias y ejes de acción variarán en función de sus fundamentos teórico-epistémicos (Hultgren, 2018; Pellow y Brehm, 2015). Algo que también sucede en el ecofeminismo que presenta muchas perspectivas que dialogan entre ellas generando un conjunto de saberes que tienen una finalidad común -despatriarcalizar los cuidados del ambiente y de las sociedades- pero no siempre acordarán los caminos para que ello sea un horizonte posible.

Si desde la ecología política se explica cómo el poder opera sobre los conflictos socioambientales a partir del estudio de los conflictos ecológicos distributivos, la ecología política feminista estudia estos conflictos desde la perspectiva feminista, fundamentando a nivel teórico y desde una corriente crítica la articulación entre ecologismo y feminismo. Se visibiliza el significativo activismo de las mujeres que denuncian los conflictos en torno a la distribución, tanto natural (clima, yacimientos de minerales, calidad del suelo, entre otros) como social (sistema político, cultural, tecnológico y económico), de recursos naturales que se constituyen como soporte de la vida (Martínez Alier, 2006, p. 104). Estas activistas advierten sobre la contaminación del aire, agua y suelos, frente a empresas y Estados, confluyendo en experiencias de resistencia que se resumen en el respeto a toda forma de vida a través de la cultura del cuidado (Agarwal, 1992; Svampa, 2015).

El ecofeminismo surge en los años setenta del siglo veinte y gana centralidad en la actualidad a la luz de la emergencia climática, haciéndose más visible y pasando a ocupar espacios cada vez más legitimados en los análisis socioambientales. A grandes rasgos, el ecofeminismo representa una sinergia entre ecologismo y feminismo que nace cuando una parte del feminismo comienza a interesarse por los cuerpos como sujetos capaces de producir vida y a cuestionar la explotación de los cuerpos y de la naturaleza como medios de vida, advirtiendo sobre la existencia de una crisis de la reproducción social (Federici, 2017, p. 119). 
Algunas autoras focalizan en el origen científico de esta corriente de pensamiento, situando como uno de los puntos de partida del mismo el análisis antropológico de Sherry Ortner en 1972 que constataba la asociación cultural de la figura de la mujer a la de la naturaleza (Puleo, 2017), mientras que otras autoras lo vinculan a las luchas por la defensa de la vida y del territorio fuera de la academia, dando más protagonismo a activistas -no provenientes de Europa o Estados Unidos- que practican el ecofeminismo aunque no se autoperciban con esa orientación (Svampa, 2015).

Los ecofeminismos dan cuenta de la vinculación entre la ecodependencia, ya apreciada por los ecologismos, y la interdependencia. Somos dependientes de la naturaleza porque utilizamos recursos y procesos que son naturales, haciendo imposible la vida -humana y no humana- al margen de ella. Y esta ecodependencia no puede ser pensada de manera aislada de la interdependencia, que es la necesidad de cuidados que tenemos como sociedades entre unas personas y otras, porque sería impensable una vida humana completamente autónoma de otra (Pérez Orozco, 2014).

Fundamentalmente los ecofeminismos develan otras realidades socioambientales que tienen relación con la discriminación de género, de raza, de clase y de territorios, las cuales están interconectadas y necesitan ser visualizadas integral y ecosistémicamente, no parcializando el diagnóstico sobre la crisis climática y ambiental focalizando el análisis en la limitación de los recursos materiales y energéticos.

En términos generales, los ecofeminismos se constituyen como corrientes críticas de pensamiento y a su vez como activismos políticos que, en primer lugar, revelan la conexión existente entre la explotación de los bienes comunes y la opresión de las mujeres en las sociedades modernas industriales (Mellor, 1997), y en segundo lugar, reclaman la ecojusticia, esto es, justicia en la apropiación y el reparto de los bienes para todas las identidades de género, clases, etnias y territorialidades. Los ecofeminismos son una propuesta de "resiliencia solidaria" (Puleo, 2019, p. 16) en tanto buscan alternativas para la vida desde la sororidad intercultural, esto es, la solidaridad entre mujeres y disidencias de diversas culturas, rechazando las epistemologías androcéntrica y antropocéntrica que hacen del punto de vista de los varones y de los hombres la referencia inequívoca hacia toda verdad.

Los ecofeminismos denuncian el sesgo patriarcal que mercantiliza la sostenibilidad y toda forma de relacionamiento, señalando las causas que subyacen a las asimetrías de poder en las sociedades. Los decoloniales hacen énfasis en la crítica a la modernidad occidental por su sesgo totalizante y pretendidamente universal, aunque exista una disputa interna en cuanto algunos reconocen la existencia de formas patriarcales en épocas premodernas (Paredes, 2008). Se distancian de la autopercepción como ecofeministas, adscribiendo a los feminismos comunitarios y populares, proponiendo el antiextractivismo como forma de detener el terricidio ${ }^{1}$ por parte de quienes explotan a la naturaleza más allá de su capacidad y para fines no relacionados con la supervivencia.

Los ecofeminismos ilustrados se basan en el principio de la calidad de vida, reconociendo los valores de la libertad y la igualdad propios de la modernidad pero con capacidad crítica de las tecnologías que afectan la dignidad y el bienestar de las especies. Se posicionan como una superación tanto del esencialismo como del relativismo cultural en tanto que critican el biologicismo desde el constructivismo y no rechazan la ciencia sino que proponen un cambio hacia un conocimiento menos androcéntrico y antropocéntrico. Muestran los sesgos de sexo, clase, raza y especie existentes en la forma de hacer ciencia, presentando como categorías centrales la sostenibilidad de la vida, entendida como parte de una ética de los cuidados (Puleo, 2017).

1 Este concepto es un aporte de Moira Millán, Weychafe de la comunidad Mapuche, que refiere a crímenes contra la vida y la tierra por parte de los Estados y de las corporaciones multinacionales que compran y expolian los territorios. 


\section{VARIEDADES DE AMBIENTALISMOS}

Los movimientos en defensa del ambiente presentan sus singularidades en función de cómo entienden la naturaleza, la sociedad y la relación entre ambas, la sostenibilidad y las causas del deterioro ambiental. En base a sus formas de actuación, definición de estrategias y posibles soluciones, existen reivindicaciones con foco en la reducción del daño ambiental por cuestiones éticas, estéticas y económicas, pero también el reclamo por el derecho a la salud pública denunciando los impactos de un medio ambiente saturado de sustancias tóxicas, e incluso existen reivindicaciones de cambio de sistema socioeconómico, remarcando la necesidad de una reconfiguración completa de las relaciones humanas y no humanas en demanda de justicia ambiental.

Un acercamiento analítico a la bibliografía científica sobre las variedades de ambientalismos ${ }^{2}$, permite distinguir entre dos grandes bloques de ambientalismos, el hegemónico y la corriente ambiental crítica (D Amico y Agoglia, 2019), en función de los diagnósticos que realizan de la realidad socioambiental, de sus propósitos y de cómo se relacionan con los actores político-institucionales y otros movimientos sociales para alcanzarlos.

Dentro del ambientalismo hegemónico destaca el paradigma del desarrollo sostenible que proviene del debate político internacional y tiene la finalidad de mitigar el deterioro ambiental con instrumentos económicos como modo de contención de reivindicaciones críticas al sistema. Por otra parte, la corriente ambiental crítica representa un conjunto de teorías más disruptivas y radicales en sus principios visibilizando las contradicciones entre capital y naturaleza (D'Amico y Agoglia, 2019, p. 98) y planteando la necesidad de un cambio del sistema de producción.

Martínez Alier (2006) realiza otra tipología dentro del ecologismo en función de las características de los movimientos ambientales, que se complementa con la distinción anterior. Observa tres corrientes dentro del ecologismo: i) "el culto de la vida silvestre": aquellos grupos preocupados por la defensa y preservación de la naturaleza en su estado original/silvestre, ii) "la ecoeficiencia": quienes depositan la confianza en la tecnología para mitigar el deterioro ambiental y iii) "el ecologismo de los pobres": resistencias locales -campesinas, indígenas- con un interés en el medio ambiente como fuente y condición para la vida sin necesariamente autopercibirse como ecologistas (Martínez Alier, 2006, p. 15). La forma en la que estas corrientes ecologistas se relacionan con las ciencias ambientales (la biología de la conservación, la ecología industrial y la ecología política), con el Estado y con otros movimientos sociales permite sostener que tanto "el culto de la vida silvestre" como "la ecoeficiencia" pertenecen al bloque del ambientalismo hegemónico mientras que "el ecologismo de los pobres" se sitúa en la corriente ambiental crítica.

Otros autores distinguen entre los grupos conservacionistas y los ecologistas de acuerdo con las diferentes maneras de entender el ambiente y la sostenibilidad, que pueden incluir o no la perspectiva de género y la justicia social. Desde esta lectura los conservacionistas trabajarán para mantener en buenas condiciones de conservación el medio natural exclusivamente dentro del contexto institucional, sin vinculación con movimientos sociales reivindicativos. Mientras que los grupos ecologistas adoptarán una actitud política y tenderán a la acción directa y otras estrategias de confrontación para intentar cambiar la realidad socioambiental (Alcock, 2008).

2 En este artículo se utilizarán los conceptos de ambientalismo y ecologismo indistintamente. No obstante, existe una diferente conceptualización entre los colectivos ecologistas y ambientalistas que proviene de la distinción entre los movimientos ecologistas del norte y los movimientos ambientalistas del sur. Para mayor detalle, se pueden consultar los textos de Enrique Leff (1998) y de Martínez Alier (2006). 
De acuerdo con la clasificación relativa a los ambientalismos hegemónicos y críticos, los grupos conservacionistas entienden que el estado y el mercado en su forma actual pueden contener la crisis ambiental implementando medidas globales, de carácter institucional y consensuadas, capaces de mitigar el deterioro ambiental. Esta perspectiva los sitúa dentro del bloque de los ambientalismos hegemónicos. Por otro lado, los grupos ecologistas adoptan una mirada crítica que se expresa en resistencias contra el proceso de mercantilización de la naturaleza que subyace a la gestión socioambiental en la actualidad, y se corresponde con los propósitos del bloque ambiental crítico.

Asimismo se observa una diferente conceptualización entre ecologismo institucional u oficial para referirse al conservacionismo y el ecologismo activista para referirse a los grupos ecologistas que tienen un propósito declarativo de modificar los modelos de producción y consumo vigentes (López Ruiz, 2013). Otra vez esta distinción se puede leer en clave de ambientalismo hegemónico (el conservacionismo, que por motivos científicos y estéticos tienen como finalidad la preservación del medio ambiente y no tienen vinculación con los feminismos, y el ecologismo institucional, que busca el desarrollo sostenible y la modernización ecológica a través de un uso razonable de los recursos) y ambientalismo crítico (un ecologismo activista con una finalidad transformadora de los sistemas de producción y acumulación).

A su vez, cada bloque ambientalista se relaciona, cómoda o incómodamente, con al menos una variedad de ecofeminismo. Si bien la mayoría de los ecofeminismos tienen postulados críticos del funcionamiento del actual sistema capitalista que mercantiliza toda forma de relacionamiento, el ambientalismo hegemónico ha incorporado una perspectiva de género, dentro de su repertorio y discursos institucionales dada la demostrada necesidad de los cuidados como tareas esenciales para garantizar condiciones de vida para el conjunto de la población.

Hay que aclarar que la perspectiva de género es una manera de abordar la realidad en función de las variables sexo y género a partir de una construcción sociohistórica y cultural de normas, estereotipos, conductas y hábitos diferentes en base a los géneros. Pero mientras que la perspectiva de género es una categoría analítica que estudia las relaciones de poder en las sociedades en función de los géneros, la perspectiva (eco)feminista incluye la interseccionalidad, la situacionalidad y la acción transformadora (Haraway, 1995; Maffía, 2005), sosteniendo que las estructuras de género son sólo una parte del total de opresiones de clase, raza, territorios y especies.

\section{Ambientalismo hegemónico: mujeres, desarrollo y medio ambiente}

Una de las banderas de los movimientos ambientalistas es caminar hacia la sostenibilidad. Desde el año 1987 a partir de la publicación del Informe Brundtland, elaborado por la Comisión Mundial de Medio Ambiente y Desarrollo de Naciones Unidas encabezada por la ex primera ministra de Noruega, Gro Harlem Brundtland, se comienza a hablar de las tres dimensiones de la sostenibilidad (la ambiental, la económica y la social) priorizando el desarrollo económico como condición de progreso para las sociedades a través de políticas que permitan un manejo racional de los recursos naturales.

Los colectivos ambientalistas que adhieren a estos postulados al trabajar dentro de las dinámicas de los organismos transnacionales entienden la sostenibilidad en su definición más clásica en tanto desarrollo sostenible que permite que las próximas generaciones puedan satisfacer sus necesidades de la misma manera que lo estamos haciendo actualmente, poniendo el énfasis en el componente económico-material de la sostenibilidad. Esta definición de la sostenibilidad proviene de la economía ambiental, 
la cual apunta a considerar los daños ambientales y sociales como externalidades que pueden ser mitigadas por ciertos mecanismos monetarios y económicos (Martínez Alier, 2006).

Dentro del bloque hegemónico destaca el paradigma del desarrollo sostenible que contiene dos variedades de ecologismos: el conservacionismo y el liberal. El conservacionismo romantiza la idea de un mundo salvaje y puro, fomenta el cuidado de la naturaleza como un valor en sí mismo (Martínez Alier, 2006, p. 16) y tiene como objetivo la protección ambiental y ecosistémica, mientras que el ambientalismo liberal tiene como propósito la defensa del ambiente y de un comercio sustentable, con el mercado como actor principal al servicio de una economía circular, es optimista en términos tecnoecológicos, e incorpora la perspectiva de género en términos institucionales.

Tanto el ecologismo conservacionista como el liberal forman parte del ambientalismo hegemónico en el sentido de ser el ambientalismo dominante porque están instalados en las percepciones de la mayoría de las personas sobre lo que se entiende por reivindicaciones ecologistas, en las agendas de la política de responsabilidad social de empresas, Estados y organismos multilaterales, así como también en las orientaciones del debate público internacional, académico y de entidades no gubernamentales. Un ambientalismo separado de la cuestión social que, desde el marco de interpretaciones occidentales y modernas del mundo (Rauchecker y Chan, 2016, p. 12) entienden la naturaleza a partir de una visión instrumental y no como condición esencial para la supervivencia. El conservacionismo la interpreta como algo prístino a preservar de la intervención humana, y el ambientalismo liberal como un conjunto de recursos y servicios ambientales definidos más por su valor de cambio que por su valor de uso (Martínez Alier, 2006).

En los ambientalismos liberales se pueden encontrar análisis y propuestas con perspectiva de género correspondientes a la perspectiva denominada "Mujeres en desarrollo" (WID -Women in Development- por sus siglas en inglés) dentro del marco de la gobernanza ambiental global, en donde "la mujer se convierte en foco de la política pública para el desarrollo como productora y agente económico, especialmente en la agricultura", hasta entonces marginada a nivel institucional en ese campo (Sturgeón, 1999, p. 266).

La cuestión de género en los discursos del ambientalismo liberal incorpora a las mujeres como un grupo homogéneo (Agüera Cabo, 2010) capaz de liderar la lucha contra la crisis socioambiental. Por tal razón, en conferencias y cumbres internacionales sobre el clima y el medio ambiente se fomenta el empoderamiento de las mujeres para resolver la problemática ambiental debido a que se asocian los valores femeninos con grados más altos de conciencia ambiental. La explicación de la distinción de valores está basada en diferencias biológicas y/o de socialización entre hombres y mujeres, en donde el sexo puede explicar los diferentes grados de sensibilidad y preocupación ambientales (Somma y Tolleson-Rinehart, 1997; Stern, Dietz y Kalof, 1993).

El uso predominante que el ambientalismo liberal hace de la perspectiva de género está basado en la adopción de políticas reformistas para incorporar a las mujeres y disidencias a los lugares de toma decisiones, luego de haber sido histórica y tradicionalmente relegadas al ámbito doméstico. Desde estas posturas se entiende al ecofeminismo como una propuesta de mejora de la realidad existente para el género femenino y para su capacidad de gestión de los recursos naturales, sin cuestionar el sistema patriarcal, esto es, la relación entre la desigualdad de género y la estructura social.

El ambientalismo liberal incorpora a su ideario, por necesidad y oportunidad, a raíz de las demandas de los feminismos, la perspectiva de género como un instrumento que le permita mantener su legitimidad, recomendando tecnocráticamente la mitigación del deterioro ambiental y considerando a las mujeres como promotoras de la modernización 
ecológica en la defensa por la naturaleza, dentro de un marco institucional en donde muchos actores interactúan para conseguir financiamiento, marcos regulatorios, gestión públicoprivada y desarrollos tecnológicos como respuesta para paliar los daños ambientales (D’Amico y Agoglia, 2019).

\section{Ambientalismo crítico: Ecofeminismos de la supervivencia, ilustrado y social}

El ambientalismo crítico está representado por un conjunto de teorías críticas que cuestionan las bases del sistema capitalista y patriarcal señalando que la crisis climática y socioambiental está relacionada con un conjunto de desigualdades estructurales: la inequidad de género, el sobreconsumo de los países del denominado "primer mundo" y el colonialismo. La crisis ambiental expresada en la sobreexplotación de los bienes naturales y la crisis social basada en la dominación y violencia contra los colectivos históricamente oprimidos y contra-hegemónicos son parte constitutiva de un mismo fenómeno. Este ambientalismo agrega la variable del poder y el análisis sistémico de la realidad social de los cuales carecían los ambientalismos liberales, que buscan la mejora del ambiente a través de un comercio sustentable. Por todo ello proponen medidas estructurales: una revisión del modelo de desarrollo vigente que valorice los cuidados con la finalidad de lograr sociedades más justas, con redistribución equitativa de los recursos y biodiversidad cultural y social (Mellor, 1997; Shiva, 1994) y otra forma de entender la sostenibilidad.

En comparación con las tradiciones reformistas de colaboración con Estados y mercados asociadas al ambientalismo hegemónico (Hultgren, 2018; Pellow y Brehm, 2015), el ambientalismo crítico valora a la naturaleza como un conjunto de bienes que dan derecho a la subsistencia de las poblaciones (Martínez Alier, 2006, p. 4). Esta cosmovisión de la naturaleza se enmarca en la "cultura del cuidado", esto es, la consideración del trabajo de la reproducción social (todo lo que no se considera trabajo productivo) a partir de la reciprocidad, la cooperación y la complementariedad, evidenciando las relaciones de poder y cuestionando "el hecho capitalista" (Svampa, 2015, p. 127).

El ambientalismo crítico entiende la sostenibilidad como transformación del sistema económico vigente que ha explotado y extraído el capital natural superando la capacidad de regeneración de los bienes naturales y produciendo residuos que no pueden ser naturalmente absorbidos (Naredo, 2010; Riechmann, 2010). Corrientes de pensamiento críticas, como la ecología política feminista y los ecofeminismos, definen la sostenibilidad como la búsqueda de la calidad ambiental y la justicia social a través de la revalorización de los cuidados para la reproducción de la vida (Pérez Prieto, 2016, p. 84).

Dentro de los ecofeminismos alineados con el bloque del ambientalismo crítico encontramos a los ecofeminismos de la supervivencia, cuyo origen se sitúa en el sur global, el ecofeminismo ilustrado y el ecofeminismo social o materialista, perspectivas provenientes del denominado norte global.

Los ecofeminismos de la supervivencia deben su nombre al riesgo de poner sus cuerpos y vidas en peligro ante la creciente violencia y militarización que el extractivismo impone en los territorios que defienden, incluidos sus cuerpos. Contra eso, proponen el fomento de valores como la reciprocidad y la cooperación en la defensa por los territorios como fuente de vida (Svampa, 2015). Desde sus vertientes, que van desde el espiritualismo al materialismo, cuestionan al ecofeminismo occidental por su visión eurocentrista que no considera las múltiples formas de vida y relacionamiento entre las comunidades y la naturaleza que presentan otras culturas y sociedades.

Los ecofeminismos de la supervivencia generalmente no se autoperciben como ecofeministas por considerar el ecofeminismo un concepto occidental (Cabnal, 2010; 
Paredes, 2008). Muchas veces las mujeres que forman parte de las luchas eco-territoriales en defensa del ambiente "no se consideran a sí mismas ni feministas ni ecologistas" (Fernández Bouzo, 2020), simplemente buscan estrategias para sobrevivir en un contexto crítico de amenaza de sus territorios. Se caracterizan por ser movimientos en donde existe un gran activismo de mujeres respondiendo de manera colectiva y no jerárquica ante las amenazas ambientales en sus hogares y comunidades, disputando a los hombres los espacios de liderazgo y toma de decisión histórica y culturalmente a ellos asignados (Maleta, 2011).

La espiritualidad de los ecofeminismos puede ser entendida desde las cosmovisiones ancestrales pero también desde la teología denunciando la dominación en las relaciones de poder en función del sexo, la raza y la religión. Los ecofeminismos espiritualistas latinoamericanos, algunos críticos del esencialismo, tienen una base en la teología feminista de la liberación que nace de la crítica a las teologías occidentales con componentes patriarcales que fueron heredadas. Mujeres religiosas progresistas que trabajan en defensa de la salud y de los territorios con comunidades empobrecidas que viven en barrios marginales (Gebara, 2002) que se autoidentifican como ecofeministas en tanto han hecho una reformulación de cómo se perciben a sí mismas en vinculación con la tierra, el cosmos, dios y diversas posturas éticas y espirituales (Ress, 2010).

En los feminismos comunitarios la opresión de género se piensa en términos de comunidad, en la que existe un principio de complementariedad entre mujeres y hombres que es jerárquico y vertical, donde los últimos son los privilegiados que subordinan a las mujeres. Por ello se propone construir una complementariedad horizontal y sin jerarquías para la armonía de la comunidad como alternativa a la sociedad individualista y patriarcal (Paredes, 2008), el respeto por el multiculturalismo y un retorno a las cosmovisiones autóctonas. Sin reivindicar la idea de una naturaleza originariamente paradisíaca, plantean la adopción de otras cosmologías que vuelvan a sacralizar la naturaleza y deslegitimicen la cosificación que el hombre hace de ella fundamentada en su pretendida superioridad.

El ecofeminismo de Vandana Shiva con componentes espiritualistas pero también materialistas, hace un llamamiento a la diversidad y el respeto por las culturas no occidentales, denunciando la mercantilización y privatización de los bienes comunes a partir de la consolidación de los poderes concentrados producto de la globalización corporativa y el neoliberalismo (2016). La autora sostiene que somos seres interdependientes, y como tales, nuestra manera de relacionarnos entre unas personas y otras y con la tierra no puede ser en términos de dominación sino de reciprocidad, respeto y cooperación, fomentando el principio productor de vida y de interconexión, vinculado a la sostenibilidad y a los cuidados. "La interconexión de toda la vida en la tierra, de los problemas y de sus soluciones, es uno de los descubrimientos más importantes de los ecofeminismos" (Mies y Shiva, 2014, p. 497).

El materialismo de los ecofeminismos de la supervivencia hace hincapié en la denuncia del colonialismo argumentando que las dinámicas de ocupación de territorios en épocas coloniales, que todavía persisten bajo otros formatos de dominación, generaron en el orden económico el despojo de bienes, minerales y energía de los territorios colonizados y, en el orden simbólico-cultural la occidentalización y la imposición de los valores eurocentristas (Bidaseca y Vázquez Laba, 2011). Estos ecofeminismos son movimientos de la praxis de defensa territorial que denuncian la subordinación de las mujeres y cuerpos feminizados, y de la naturaleza a la supremacía patriarcal. Son formas de conocimiento vinculadas a prácticas ambientalistas territoriales lideradas por activistas, que disputan el sentido y la racionalidad de la colonial-modernidad occidental euronorteamericana.

El ecofeminismo ilustrado, por su parte, tiene como base los principios de igualdad, libertad y sostenibilidad, proponiendo la razón ilustrada como fuerza analítica, crítica 
de los dogmas y de toda forma de patriarcado, prescindiendo de la mística y de la fe en algo trascendente. En línea con los postulados del movimiento ilustrado, se trata de un ecofeminismo "que no caiga en la habitual renuncia al universalismo ilustrado y a sus ideales reguladores" (Puleo, 2008, p. 42), que sea capaz de vincular los derechos humanos con la justicia ambiental y la ecoética. Su objetivo es trasladar su proyecto de emancipación universal al conjunto de la ciudadanía, reivindicando la igualdad de género y la ética de los cuidados de la naturaleza, de las personas y de los animales no humanos, aceptando moderadamente la ciencia y la técnica como parte del proyecto de progreso evolutivo (Puleo, 2008).

La corriente ecofeminista ilustrada es una postura filosófica que tiene como punto de partida la crítica del antropocentrismo y el sesgo masculino que presenta la cultura androcéntrica y propone reelaborar los términos de humanidad y naturaleza. Por tanto, se presenta como "una alternativa a la globalización neoliberal androantropocéntrica" (Puleo, 2019, p. 42), aconsejando no esencializar el rol de la mujer en su vinculación con la naturaleza por una cuestión biologicista, y sobre todo, "desgenerizar" y universalizar los trabajos de cuidados, esto es, que sean responsabilidad de toda la ciudadanía, independientemente del sexo (Puleo, 2011, p. 274). Desde la corriente ilustrada se sostiene que actualmente feminismo y ecologismo, ambas teorías que surgen de la ilustración, son "dos mundos que viven de espaldas pero que en el futuro están destinados a tratarse y, probablemente, a realizar pactos políticos" (Puleo, 2002, p. 37).

El ecofeminismo social o materialista denuncia que el deterioro socioambiental es producto del sistema capitalista -cuya base es la división sexual del trabajo- y la estructuración social desigual que pone a la mujer en el lugar de la reproducción, al hombre en el ámbito de la producción y a la naturaleza al servicio del hombre (Mellor, 1997). Patriarcado y capitalismo constituyen una alianza histórica de dominación y explotación del cuerpo femenino y de la naturaleza en tanto las relaciones de género son relaciones de poder que legitiman el funcionamiento de los actuales modos de producción, consumo y reproducción (Aguinaga, 2010; Arruzza, 2016).

La corriente ecofeminista social estudia el tratamiento de las mujeres como colonia interna de los Estados Nacionales, donde tanto el trabajo doméstico que éstas realizan como los costes medioambientales de la actividad económica son considerados como "externalidades" del sistema de producción, y esto supone que ambos se convierten en los refuerzos del sistema capitalista patriarcal (Federici, 2017; Pérez Orozco, 2014). Esto significa y de acuerdo con Silvia Federici (2017), que la nueva acumulación del capital se realiza en función de dos cuestiones: el trabajo gratuito e invisibilizado que como actividad desempeñan las mujeres en el ámbito doméstico y los bienes y recursos naturales que nos provee la naturaleza para el funcionamiento de la vida.

\section{REFLEXIONES FINALES}

En el mundo actual bajo la crisis socio-ecológica global que estamos atravesando se ha puesto en evidencia la importancia de los cuidados y de los trabajos reproductivos, que tradicional e históricamente han recaído sobre las mujeres y cuerpos feminizados. La defensa ambiental desde una perspectiva feminista se hace más necesaria que nunca para cambiar el rumbo en una dirección deseada. Con esta intención se ha presentado el conjunto de las principales teorías desde las que se puede abordar el estudio de los movimientos ambientalistas en relación con sus variedades y con el feminismo, prestando especial atención en los ecofeminismos. 
Dentro de la bibliografía de la sociología ambiental los feminismos aportan a los ambientalismos una narrativa ecofeminista que es cada vez más frecuente y legitimada empírica y teóricamente. Pero el encuentro entre ambientalismos y ecofeminismos tiene sus matices en función de los usos y apropiaciones que los ecologismos hagan de los ecofeminismos, de sus conceptos, epistemes y prácticas asociadas para su reinvención.

Considerando a los ecofeminismos como narrativas necesarias para reinterpretar la cuestión socioambiental y darles sustancia a los colectivos ecologistas, algunos de los cuales han ido perdiendo la centralidad que tuvieron en otras épocas, abordamos en este artículo los ambientalismos y ecofeminismos como una unidad. Hemos trazado puentes y encuentros entre unas tipologías de ambientalismos y las variedades de ecofeminismos para, de esta manera, justificar la simbiosis existente entre ambos movimientos frente a la emergencia socioambiental.

Los dos grandes bloques conceptuales que representan formas de interpretar la realidad socioambiental: el ambientalismo hegemónico y el ambientalismo crítico, son enfoques que agrupan y explican las diferentes perspectivas ideológicas de los ecologismos. A partir de ellos, hemos buscado compatibilidades entre ecofeminismos y ambientalismos en función de sus entendimientos de la cuestión socioambiental, las ideas de mejora o transformación del sistema de consumo y producción vigente, las medidas a ser adoptadas para conseguir dichas finalidades, y las alianzas o relaciones con otros movimientos sociales, comunidad científica y Estados.

No todas las tipologías de ambientalismos se corresponden con una variedad de ecofeminismo y en algunos casos, existe una afinidad meramente instrumental. Tal es el caso del bloque del ambientalismo hegemónico que contiene al conservacionismo el cual no tiene relación con el movimiento feminista, y el ambientalismo liberal que aplica la perspectiva de género a la cuestión ambiental, presentando a las mujeres como nuevas protagonistas para la resolución de la problemática ambiental siempre que sus ideas no vayan más allá de los límites de los dogmas de la ideología productivista y economicista dominante. Por su parte, el bloque ambiental crítico contiene al ecologismo social y activista que agrupa en su interior expresiones de los ecofeminismos de la supervivencia, del Sur Global, como al ecofeminismo ilustrado y al ecofeminismo materialista, ambos del Norte Global.

Que una corriente de pensamiento sea hegemónica o crítica tiene relación con su mayor o menor grado de permeabilidad social, accesibilidad y legitimación, razón por la cual la primera terminará dominando y marginando a la segunda. Pero la problemática socioambiental no sólo es una cuestión teórica sino también empírica y, así como las epistemologías dan sustento y fundamento a las prácticas sociales (Walter, 2009) también la praxis social puede cambiar paradigmas y teorías dominantes. Por ello sigue resultando necesario continuar estudiando los movimientos en defensa de la cuestión socioambiental desde las prácticas que llevan adelante las personas que los integran en materia de cuidados, que es una de las categorías teóricas centrales de los ecofeminismos.

Proponer horizontes social y ecológicamente deseables y posibles disputa la apropiación, esterilización, neutralización y despolitización de la cuestión ambiental por parte de sectores de poder que buscan la acumulación de ganancias y la continuidad del status quo. Esas ideas actualmente hegemónicas no son dogmas irreemplazables, tienen un sustento social que no es eterno y es pasible de transformación. Por ello, visibilizando, desde instancias comunitarias, de base y académicas, la oportunidad que tienen los ecologismos sociales y populares de incorporar estas narrativas, se construye hegemonía para activar la capacidad crítica y de lucha contra el deterioro ambiental y la polarización social y territorial. 
En un contexto en el cual, afortunadamente, los feminismos ganan espacio en las agendas públicas y los ecologismos clásicos se reestructuran, en donde coexisten viejas y nuevas formas que están emergiendo de la mano de las nuevas generaciones, se trata de articular nuevas formas de pensar, sentir y hacer para ir generando mayor receptividad social hacia la revisión crítica de lo establecido.

\section{REFERENCIAS}

Agarwal, B. (1992). The Gender and Environment Debate: Lessons from India. Feminist Studies, 18, 119-158. https://doi.org/10.2307/3178217

Agüera Cabo, M. (2010). Poder y género en la lucha ambiental. Casos de estudio en el norte de Cataluña. Investigaciones Feministas, 1, 9-26.

Aguinaga, M. (14 de julio de 2010). Ecofeminismo: mujer y Pachamama, no solo es posible una crítica al capitalismo y al patriarcado. América Latina en movimiento [en línea]. Recuperado de https://www.alainet.org/es/active/39531

Alcock, F. (2008). Conflicts and Coalitions Within and Across the ENGO Community. Global Environmental Politics, 8, 66-91. https://doi.org/10.1162/glep.2008.8.4.66

Alimonda, H. (2006). Paisajes del Volcán de Agua. Aproximación a la ecología política latinoamericana. Gestión y Ambiente, 9, 45-54.

Arruzza, C. (3 de julio de 2016). Reflexiones sobre el género. ¿Cuál es la relación entre el patriarcado y el capitalismo? [en línea]. Sin permiso. Recuperado de https://sinpermiso. info/textos/reflexiones-sobre-el-genero-cual-es-la-relacion-entre-el-patriarcado-yel-capitalismo-se-reabre-el

Bidaseca, K. y Vázquez Laba, V. (2011). Feminismos y poscolonialidad: Descolonizando el feminismo desde y en América Latina. Buenos Aires: Godot.

Cabnal, L. (2010). Acercamiento a la construcción de la propuesta de pensamiento epistémico de las mujeres indígenas feministas comunitarias de Abya Yala [en línea]. ACSUR. Recuperado de https://porunavidavivible.files.wordpress.com/2012/09/feminismoscomunitario-lorena-cabnal.pdf

Connell,R.(1990). Awholenewworld:Remakingmasculinityin the context of the environmental movement. Gender and Society, 4, 452-478. https://doi.org/10.1177/089124390004004003

D’Amico, P. y Agoglia, O. (2019). La cuestión ambiental en disputa: el ambientalismo hegemónico y la corriente ambiental crítica. Lecturas desde y para América Latina. Revista Colombiana de Sociología, 42, 97-116. https://doi.org/10.15446/rcs.v42n1.73247

Federici, S. (2017). Diálogos entre el feminismo y la ecología desde una perspectiva centrada en la reproducción de la vida. Ecología política, 54, 119-122.

Fernández Bouzo, S. (2020). Imaginaciones socio-ecológicas. Apuntes para ensayar mundos con justicia ecosocial. Revista Florestanía. Recuperado de http://www.florestania. com.ar/2020/06/05/imaginaciones-socioecologicas-apuntes-para-ensayar-mundoscon-justicia-ecosocial/?ffbclid=IwAR2ib1CaS50Yd-mdY_AP3JbgBUem4xe8L6iA5U_ GRwRh_2m5a0qCg1ZCir0

Gebara, I. (2002). Diez años de Con-spirando. Revista Latinoamericana de Ecofeminismo, Espiritualidad y Teología, 40, 3-14. 
Haraway, D. (1995). Ciencia, cyborgs y mujeres. La reinvención de la naturaleza. Madrid: Ediciones Cátedra.

Herrero, Y. (4 de julio de 2016). El ecofeminismo habla de la política de lo cotidiano [en línea]. El diari de l'escola d'estiu. Recuperado de http://diarieducacio.cat/ escolaestiurosasensat/2016/07/04/yayo-herrero-ecofeminismo-habla-de-la-politicade-lo-cotidiano/

Hultgren, J. (2018). 21st century American environmental ideologies: A reevaluation. Journal of Political Ideologies, 23, 54-79. https://doi.org/10.1080/13569317.2017.1397916

Intergovernmental Panel on Climate Change. (2014). Synthesis Report: Climate Change. Recuperado de https://www.ipcc.ch/report/ar5/syr/

Leff, E. (1998). Saber ambiental. Sustentabilidad, racionalidad, complejidad, poder. México: Siglo XXI.

López Ruiz, J. (2013). El ecologismo político en España: De la crisis ecológica a la acción política (Tesis de doctorado). Universidad de Valencia, Facultad de Derecho, Departamento de Filosofía del Derecho, Moral y Política, Valencia.

Maffía, D. (2005). Epistemología feminista: por otra inclusión de los femenino en la ciencia. En N. Blázquez Graf y J. Flores (ed.), Ciencia, tecnología y género en Iberoamérica (pp. 623633). México: Centro de Investigaciones Interdisciplinarias en Ciencias y Humanidades.

Maleta, Y. (2011). Social dimensions of gender and hegemony within environmental organisations and communities. The International Journal of Diversity in Organisations, Communities and Nations, 10, 79-91. https://doi.org/10.18848/1447-9532/CGP/ v10i06/38945

Martínez Alier, J. (2006). El ecologismo de los pobres: conflictos ambientales y lenguajes de valoración. Barcelona: Icaria.

Mellor, M. (1997). Feminismo y Ecología. Nueva York: University Press.

Mies, M. y Shiva, V. (2014). Ecofeminism. London: Zed Books.

Naredo, J. M. (2010). Raíces económicas del deterioro ecológico y social. Más allá de los dogmas. Madrid: Siglo XXI.

Paredes, J. (2008). Hilando fino desde el feminismo comunitario. Recuperado de https:// mujeresdelmundobabel.org/files/2013/11/Julieta-Paredes-Hilando-Fino-desde-elFem-Comunitario.pdf

Pellow, D. N. y Brehm, H. N. (2015). From the New Ecological Paradigm to Total Liberation: The Emergence of a Social Movement Frame. Sociological Quarterly, 56, 185-212. https://doi.org/10.1111/tsq.12084

Pérez Orozco, A. (2014). Crisis multidimensional y ajuste feminizado: retos y oportunidades. En C. Carrasco Bengoa (ed.), Con voz propia. La economía feminista como apuesta teórica y política (pp. 171-192). Madrid: La oveja roja.

Pérez Prieto, L. (2016). La sostenibilidad de la vida humana y no humana. Una aproximación feminista y ecologista a las ciudades (Tesis de doctorado). Universidad Pablo de Olavide, Departamento de Economía, Métodos Cuantitativos e Historia Económica, Sevilla.

Puleo, A. (2002). Feminismo y ecología. El ecologista, 31, 36-39. 
Puleo, A. (2008). Libertad, igualdad, sostenibilidad. Por un ecofeminismo ilustrado. Isegoría. Revista de Filosofía Moral y Política, 38, 39-59. https://doi.org/10.3989/isegoria.2008. i38.402

Puleo, A. (2011). Ecofeminismos para otro mundo posible. Madrid: Cátedra.

Puleo, A. (2017). Perspectivas ecofeministas de la ciencia y el conocimiento. La crítica al sesgo andro-antropocéntrico. Daimon. Revista internacional de filosofía, 6, 41-54. http://dx.doi.org/10.6018/daimon/290751

Puleo, A. (2019). Claves ecofeministas. Para rebeldes que aman a la Tierra y a los animales. Madrid: Plaza y Valdés.

Rauchecker, M. y Chan, J. (2016). Las luchas por la sustentabilidad desde abajo: hacia una nueva agenda de investigación. En M. Rauchecker y J. Chan (eds.), Sustentabilidad desde abajo. Luchas desde el género y la etnicidad (pp. 11-30). Buenos Aires: CLACSO.

Ress, M. J. (2010). Espiritualidad ecofeminista en América Latina. Investigaciones Feministas, 1, 111-124. Recuperado a partir de https://revistas.ucm.es/index.php/INFE/article/view/ INFE1010110111A

Riechmann, J. (2010). Biomímesis. Ensayos sobre imitación de la naturaleza, ecosocialismo y autocontención. Madrid: Los libros de la catarata.

Shiva, V. (1994). Close to Home: Women Reconnect Ecology, Health and Development Worldwide. Londres: Earthscan.

Shiva, v. (2016). Who really feeds the world. The failures of Agribusiness and the promise of Agroecology. North Atlantic Books.

Somma, M. y Tolleson-Rinehart, S. (1997). Tracking the elusive green women: Sex, environmentalism, and feminism in the United States. Political Research Quarterly, 50, 153-169. https://doi.org/10.1177/106591299705000108

Stern, P. C., Dietz, T. y Kalof, L. (1993). Value Orientations, Gender, and Environmental Concern. Environment and Behavior, 25, 322-348. https://doi.org/10.1177/0013916593255002

Sturgeon, N. (1999). Ecofeminist appropriations and transnational environmentalisms. Identities Global Studies in Culture and Power, 6(2-3), 255-279. https://doi.org/10.1080/ 1070289X.1999.9962645

Svampa, M. (2015). Feminismos del Sur y Ecofeminismo. Revista Nueva Sociedad, 256, 127-131.

Walter, P. (2009). Philosophies of adult environmental education. Adult Education Quarterly, 60, 3-25. https://doi.org/10.1177/0741713609336109

Warren, K. (1996). Ecological Feminist Philosophies. Indiana: University Press. 


\section{NOTA BIOGRÁFICA}

M. Eugenia Mediavilla, doctoranda del Programa de Doctorado en Medio Ambiente y Sociedad, becaria colaboradora del Centro de Estudios de Posgrado e integrante del Grupo de investigación EcoEcoFem (SEJ 507) de la Universidad Pablo de Olavide, Sevilla, España. Licenciada en Ciencia Política (Universidad de Buenos Aires, Argentina) y Máster en Educación Ambiental (Universidad de Córdoba, España). Además colaboro con el grupo de investigación "Las experiencias de acción colectiva ecofeministas territoriales y su impacto en la cuestión ambiental en Argentina" y mis principales áreas de interés son los ecofeminismos populares del Sur Global.

José M. Echavarren es profesor contratado doctor en el departamento de Sociología de la Universidad Pablo de Olavide (Sevilla, España). Doctor en Sociología (Universidad Pública de Navarra, 2004), su tesis doctoral fue galardonada en el XXII Premio de tesis doctorales del Colegio Nacional de Politólogos y Sociólogos (2007). Ha trabajado como investigador postdoctoral asociado a dos proyectos del V y VI Programa Marco Europeo en el Institut de Ciència i Tecnologia Ambientals (ICTA) de la Universidad Autónoma de Barcelona. Trabajó como investigador ayudante en el Centro de Estudios Andaluces. Visiting Associate de la Universidad de York, ha publicado extensamente sobre aspectos relativos al medio ambiente en revistas internacionales y nacionales. Se le puede localizar en jmechavarren@ upo.es. Más información en www.sociologico.es 